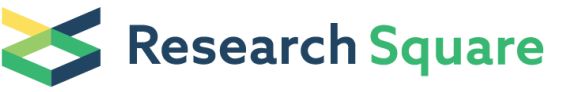 \\ Preprints are preliminary reports that have not undergone peer review. \\ They should not be considered conclusive, used to inform clinical practice, or referenced by the media as validated information.
}

\section{Longitudinal study of a SARS-CoV-2 infection in an immunocompromised patient with X-linked agammaglobulinemia}

\section{Laura Ciuffreda}

Hospital Universitario N.S. de Candelaria

José M. Lorenzo-Salazar

Instituto Tecnológico y de Energías Renovables

Julia Alcoba-Florez

Hospital Universitario N.S. de Candelaria

Héctor Rodriguez-Pérez

Hospital Universitario N.S. de Candelaria

Helena Gil-Campesino

Hospital Universitario N.S. de Candelaria

Antonio Íñigo-Campos

Instituto Tecnológico y de Energías Renovables

Diego García-Martínez de Artola

Hospital Universitario N.S. de Candelaria

Agustín Valenzuela-Fernández

Universidad de La Laguna

Marcelino Hayek-Peraza

Hospital Universitario N.S. de Candelaria

\section{Susana Rojo-Alba}

Hospital Universitario Central de Asturias

Marta Elena Alvarez-Argüelles

Hospital Universitario Central de Asturias

Oscar Díez-Gil

Hospital Universitario N.S. de Candelaria

Rafaela González-Montelongo

Instituto Tecnológico y de Energías Renovables

Carlos Flores ( $\nabla$ cflores@ull.edu.es )

Hospital Universitario N.S. de Candelaria https://orcid.org/0000-0001-5352-069X 
Keywords: Immunocompromised patient, long-term viral shedding, SARS-CoV-2, COVID-19, viral variants.

Posted Date: July 13th, 2021

DOI: https://doi.org/10.21203/rs.3.rs-702594/v2

License: (c) (1) This work is licensed under a Creative Commons Attribution 4.0 International License. Read Full License

Version of Record: A version of this preprint was published at Journal of Infection on July 1st, 2021. See the published version at https://doi.org/10.1016/j.jinf.2021.07.028. 


\section{Abstract}

We describe the case of a 23-year-old immunocompromised male patient with clinically diagnosed Xlinked agammaglobulinemia who was admitted to the hospital on the 14th April 2020 due to coronavirus disease of 2019 (COVID-19). Despite COVID-19 test negativizations, the patient was hospitalized most of the time and finally admitted to the intensive care unit where he died from multiorgan failure and shock. Over 149 days, 26 respiratory samples were collected, subjected to viral genome sequencing, and all assigned to the same lineage, supporting a single viral infection event. The accumulation of mutations throughout the course of the infection was accelerated and suggested the presence of compartmentalized viral subpopulations that evolved independently in the upper and lower respiratory airways. These results support that long-term viral shedding in immunocompromised patients is one possible mechanism for the emergence of variants of concern and provide evidence towards the infection control guidelines in these patients.

\section{Main Text}

A 23-year-old immunocompromised male patient with clinically diagnosed X-linked agammaglobulinemia (XLA) (Supplementary Material), who was treated monthly with intravenous immunoglobulin, was admitted to the hospital on the $14^{\text {th }}$ April 2020 with left bilobar pneumonia likely due to coronavirus disease 2019 (COVID-19). At the time of admission, the patient reported cough and chronic diarrhoea, fever over the last four days, and a nasopharyngeal (NP) swab sample testing positive for Severe Acute Respiratory Syndrome Coronavirus 2 (SARS-CoV-2) by RT-qPCR (Figure 1A). The patient was immediately treated with antibiotics (azithromycin, ceftazidime) and compassionate use of hydroxychloroquine (12day course). On day 16 , four days after the end of the hydroxychloroquine treatment, the RT-qPCR assay on NP swab was negative. On day 18 , the assay was positive again turning back negative three days later. From day 21 to 44, RT-qPCR assays of five NP swab samples were negative for SARS-CoV-2. On day 24 , the patient developed respiratory symptoms, including cough, and, following computed tomography (CT), he was diagnosed with viral pneumonia with bacterial superinfection. Despite clinical findings, no microbial pathogens were isolated from the bronchoalveolar lavage (BAL) samples. He was given another course of antibiotics (meropenem, linezolid and co-trimoxazole (a combination of trimethoprim and sulphamethoxazole)), antifungal treatment (amphotericin B), and intravenous glucocorticoids (methylprednisolone). On day 31, following the detection of SARS-CoV-2 in a BAL sample (day 29), the patient received the antiviral drug remdesivir for compassionate use (10-day course).

On day 45, since he was asymptomatic and had a negative RT-qPCR from a NP swab sample suggestive of a resolved infection, he was discharged from the hospital. However, the following day, he returned to the hospital reporting fever and bleeding from his right ear. He was again admitted to the hospital and tested for SARS-CoV-2 due to concern for a possible COVID-19 recurrence. The RT-qPCR of a NP swab sample was positive on day 47. From this moment onwards, the patient was persistently positive for SARS-CoV-2 infection. Infectious SARS-CoV-2 was also successfully cultured from a BAL sample on day 50 , showing that the virus was actively replicating in the lower respiratory airways of the patient 
(Supplementary Material). The patient was given another course of antibiotics (cefepime, meropenem, linezolid, piperacillin/tazobactam, and prophylactic co-trimoxazole), antifungal treatments (amphotericin $B$, voriconazole), intravenous (methylprednisolone, dexamethasone) and oral (prednisone, dexamethasone) corticosteroids, and a second course of remdesivir (8-day course from day 103). Since no clinical improvement was observed one week after the remdesivir antiviral treatment, he was given a cocktail of the HIV (human immunodeficiency virus) protease inhibitors lopinavir/ritonavir in addition to ceftazidime, a third-generation cephalosporin beta-lactam antibiotic. No other pathogens were detected from respiratory samples up to this moment (Supplementary Material). On day 128 , given continuous respiratory decline characterised by dyspnea and tachypnea, the patient was admitted to the intensive care unit (ICU) (Supplementary Figure 1). On day 131, he was administered hyperimmune serum from a COVID-19 convalescent donor. On day 133, after many desaturation events despite high-flow nasal cannula therapy, the patient was provisioned with non-invasive mechanical ventilation. On the same day, Pseudomonas aeruginosa was isolated in a throat swab from multidrug-resistant surveillance cultures and the patient received treatment with ceftolozane/tazobactam. Three days later, he underwent endotracheal intubation because of respiratory failure. From this moment onwards, the patient was sedated and mechanically ventilated. On day 147 , since blood culturing showed the growth of a Gramnegative pathogen, which was later identified as being multidrug-resistant $P$. aeruginosa, he was given colistin (polymyxin E) and meropenem antibiotics, as a last-resort treatment. On day 149 ( $10^{\text {th }}$ September 2020), he died from multiorgan failure and shock.

Throughout the period described, 26 respiratory samples were collected from the patient (22 NP swabs and 4 BAL samples) (Figure 1A). Whole SARS-CoV-2 viral genomes of a subset of $13 \mathrm{NP}$ and $3 \mathrm{BAL}$ samples were sequenced using either Illumina or Oxford Nanopore Technologies sequencing (Supplementary Material). A urine, faeces, and peripheral blood sample (on day 44), and an additional peripheral blood sample (on day 87) were collected, but viral genome was not detected in any of their RNA extractions. All genome sequences were assigned to the PANGO lineage A.2 (Clade 19B), which was predominant in Spain during the early months of the pandemic [1]. Assignation of the sequences to the same lineage suggests that the patient had a single viral infection event. Synonymous and nonsynonymous mutations accumulated throughout the course of the infection in NP swab and BAL samples (Spearman correlation, $r=0.77, p=0.00072$ ) (Figure 1B). Different constellations of mutations were observed in the sequences isolated from NP swab and BAL samples, suggesting the presence of compartmentalized viral subpopulations that evolved independently, as was previously suggested [2]. The median mutation rate, calculated as the accumulated mutations per day since diagnosis, was 0.09 mutations/day, higher than the one originally estimated for SARS-CoV-2 (0.06 mutations/day [3]) (Onesample Wilcoxon test, $p=0.005)$, indicating accelerated mutation rate during the infection. There was no significant difference in the mutation rate calculated for NP swab and BAL samples in the infection period (Mann-Whitney test, $p=0.18$ ).

On day 0 , viral genome sequences harboured the characteristic mutational pattern of lineage A.2 (ORF1a:F3701Y, ORF3a:G196V, ORF8:L84S, N:S197L) in addition to two other substitutions and four 
synonymous mutations (Figure 1B). In particular, the spike (S) gene sequence was characterized by the I197V substitution and one synonymous mutation. These were the only two mutations observed in the $S$ gene throughout the course of this five-month period infection in the NP swab samples. However, we observed a different evolutionary pattern in the BAL samples. On day 50, the A653V amino acid substitution was observed. This has been associated as part of the mutational pattern of two variants spreading in France [4] and Germany [5] at the beginning of 2021. On day 87, the P384L in the receptorbinding domain (RBD) emerged but disappeared together with the A653V on day 136 , five days after treatment with the hyperimmune serum from a convalescent patient, when R158S and N501T substitutions emerged. Strikingly, the N501T mutation is associated with an increased binding affinity of the $S$ protein to the human angiotensin-converting enzyme 2 (ACE2) receptor and has been recently identified as an escape mutation against several anti-SARS-CoV-2 neutralizing antibodies (NAbs) (e.g., B38, CC12.1, and S309 S2H12 S304, among others) [6]. Interestingly, the position R158 of the S protein is part of the N-terminal domain (NTD) antigenic supersite, a region being recognized by all known NAbs directed to the NTD [7], and the R158S substitution has been included among the escape mutations of anti-SARS-CoV-2 monoclonal NAbs targeting the NTD of the viral S protein [8]. Besides the S gene, we highlight the emergence, on day 50 , of the G204R in the nucleocapsid (N) gene, a mutation characteristic of the P. 2 lineage, and, on day 136, of the K1795Q in the ORF1a and the P67S in the N gene, which are distinctive signatures of P.1 and B.1.617.3 lineages, respectively. P67S was also reported in the 20C-US emerging variant within the B.1.2 lineage [9].

This study describes the case of an XLA-immunocompromised patient with prolonged SARS-CoV-2 infection, supporting evidence that these patients undergo viral shedding for long periods of time, which can last up to several months [9-12]. The patient presented RT-qPCR negative NP swab samples in different time intervals throughout the course of infection that either matched to a positive BAL sample or were followed by a positive RT-qPCR sample. This indicates that a negative RT-qPCR result in NP swab samples, which are the specimens usually adopted for diagnosis and surveillance, may not imply remission from infection, as was previously observed [10-12]. Viral genome sequencing of NP and BAL samples revealed an accelerated intra-host viral evolution, as previously described in immunocompromised patients [10]. Different mutations were accumulated in samples collected from NP swabs and BAL throughout the course of infection, which may point to differential viral adaptation to the upper and the lower respiratory airways. Several host factors could account for this phenomenon, such as temperature and immune response disparities and/or tissue differences in the ACE2 expression. In fact, the upper respiratory tract shows lower temperature and type-I IFN-mediated immune responses compared to those observed in the lower respiratory tract [13], together with a higher density of the ACE2 [14]. These factors could favour viral infection and replication in the upper respiratory tract, contributing to the intra-host viral diversity in patients. Furthermore, it is important to note that the mutations emerging in the viral population present in the lower respiratory tract are not detected by sequencing NP swab samples, highlighting that the emergence of potentially worrying viral variants may be underestimated by sequencing standard diagnostics NP samples. We observe the remarkable appearance of amino acid substitutions linked to immune evasion in the BAL sample collected three days after treatment with 
hyperimmune serum. Of note, the appearance of the same or other mutations of interest in the NP swab samples days after hyperimmune serum treatment could not be ruled out. In fact, we were able to sequence only one NP swab sample $24 \mathrm{~h}$ after treatment, which may not be enough time to observe a possible viral population shifting in these samples. One limitation of our study is that we have no data on the Abs composition and SARS-CoV-2 neutralizing activity of the hyperimmune serum used. Lastly, the emergence of mutations distinctive of currently circulating SARS-CoV-2 variants of concern (VOCs) support the hypothesis for long-term viral shedding in immunocompromised patients as one possible mechanism for the emergence of VOCs.

\section{Declarations}

\section{Acknowledgements}

We deeply acknowledge the University Hospital Nuestra Señora de Candelaria (HUNSC) and the Instituto Tecnológico y de Energías Renovables (ITER) board of directors for their strong support and assistance in accessing diverse resources used in the study.

\section{Conflicts of interest}

The authors declare that they have no known competing financial interests or personal relationships that could have appeared to influence the work reported in this paper.

\section{Ethical approval}

The University Hospital Nuestra Señora de Candelaria (Santa Cruz de Tenerife, Spain) review board approved the study (ethics approval number: CHUNSC_2020_24).

\section{Funding}

This work was supported by Cabildo Insular de Tenerife [grants CGIEU0000219140 and "Apuestas científicas del ITER para colaborar en la lucha contra la COVID-19"]; the agreement with Instituto Tecnológico y de Energías Renovables (ITER) to strengthen scientific and technological education, training research, development and innovation in Genomics, Personalized Medicine and Biotechnology [grant number OA17/008]; Instituto de Salud Carlos III [grant numbers FI18/00230 and PI20/00876] and Ministerio de Ciencia e Innovación [grant numbers RTI2018-093747-B-100 and RTC-2017-6471-1], co- 
funded by the European Regional Development Fund (ERDF), "A way of making Europe" from the European Union; Lab P2+ facility [grant number UNLL10-3E-783], co-funded by the ERDF and "Fundación

CajaCanarias"; and the Spanish HIV/AIDS Research Network [grant number RIS-RETIC, RD16/0025/0011], co-funded by Instituto de Salud Carlos III and by the ERDF; and RIS-3 Canarias Strategy - "María del Carmen Betancourt y Molina" Program, "Consejería de Economía, Conocimiento y Empleo, Gobierno de Canarias" [grant number ProlD2020010093]. The funders had no role in the study design, collection, analysis and interpretation of data, in the writing of the manuscript or in the decision to submit the manuscript for publication.

\section{References}

1. Latif AA, Mullen JL, Alkuzweny M, et al. A.2 Lineage Report. 2021. Available at: https://outbreak.info/situation-reports?pango=A.2. Accessed 21 June 2021.

2. Kemp SA, Collier DA, Datir RP, et al. SARS-CoV-2 evolution during treatment of chronic infection. Nature 2021; :1-10. Available at: http://www.ncbi.nlm.nih.gov/pubmed/33545711. Accessed 17 February 2021.

3. Harvey WT, Carabelli AM, Jackson B, et al. SARS-CoV-2 variants, spike mutations and immune escape. Nat Rev Microbiol 2021; 19:409-424. Available at: https://doi.org/10.1038/s41579-021-00573-0.

4. Colson P, Levasseur A, Delerce J, et al. Spreading of a new SARS-CoV-2 N501Y spike variant in a new lineage. Clin Microbiol Infect 2021; Available at: https://doi.org/10.1016/j.cmi.2021.05.006.

5. Mallm J-P, Bundschuh C, Kim H, et al. Local emergence and decline of a SARS-CoV-2 variant with mutations L452R and N501Y in the spike protein. medRxiv 2021; Available at:

https://www.medrxiv.org/content/early/2021/04/29/2021.04.27.21254849.

6. Wang R, Chen J, Gao K, Wei G-W. Vaccine-escape and fast-growing mutations in the United Kingdom, the United States, Singapore, Spain, India, and other COVID-19-devastated countries. Genomics 2021; 113:2158-2170. Available at:

https://www.sciencedirect.com/science/article/pii/S0888754321001798.

7. McCallum M, De Marco A, Lempp FA, et al. N-terminal domain antigenic mapping reveals a site of vulnerability for SARS-CoV-2. Cell 2021; 184:2332-2347.e16. Available at:

https://doi.org/10.1016/j.cell.2021.03.028.

8. Suryadevara N, Shrihari S, Gilchuk P, et al. Neutralizing and protective human monoclonal antibodies recognizing the N-terminal domain of the SARS-CoV-2 spike protein. Cell $2021 ; 184: 2316-$ 2331.e15. Available at: https://doi.org/10.1016/j.cell.2021.03.029.

9. Pater AA, Bosmeny MS, Barkau CL, et al. Emergence and Evolution of a Prevalent New SARS-CoV-2 Variant in the United States. bioRxiv 2021; :2021.01.11.426287. Available at: 
http://biorxiv.org/content/early/2021/01/19/2021.01.11.426287.abstract.

10. Choi B, Choudhary MC, Regan J, et al. Persistence and Evolution of SARS-CoV-2 in an Immunocompromised Host. N Engl J Med 2020; 383:2291-2293. Available at: http://www.nejm.org/doi/10.1056/NEJMc2031364. Accessed 17 February 2021.

11. Baang JH, Smith C, Mirabelli C, et al. Prolonged Severe Acute Respiratory Syndrome Coronavirus 2 Replication in an Immunocompromised Patient. J Infect Dis 2021; 223:23-27. Available at: https://doi.org/10.1093/infdis/jiaa666.

12. Mendes-Correa MC, Ghilardi F, Salomão MC, et al. SARS-CoV-2 shedding, infectivity and evolution in an immunocompromised adult patient. medRxiv $2021 ;: 2021.06 .11 .21257717$. Available at: http://medrxiv.org/content/early/2021/06/15/2021.06.11.21257717.abstract.

13. V'kovski P, Gultom M, Kelly JN, et al. Disparate temperature-dependent virus-host dynamics for SARS-CoV-2 and SARS-CoV in the human respiratory epithelium. PLOS Biol 2021; 19:e3001158. Available at: https://doi.org/10.1371/journal.pbio.3001158.

14. Hou YJ, Okuda K, Edwards CE, et al. SARS-CoV-2 Reverse Genetics Reveals a Variable Infection Gradient in the Respiratory Tract. Cell 2020; 182:429-446.e14. Available at: https://doi.org/10.1016/j.cell.2020.05.042.

\section{Figures}




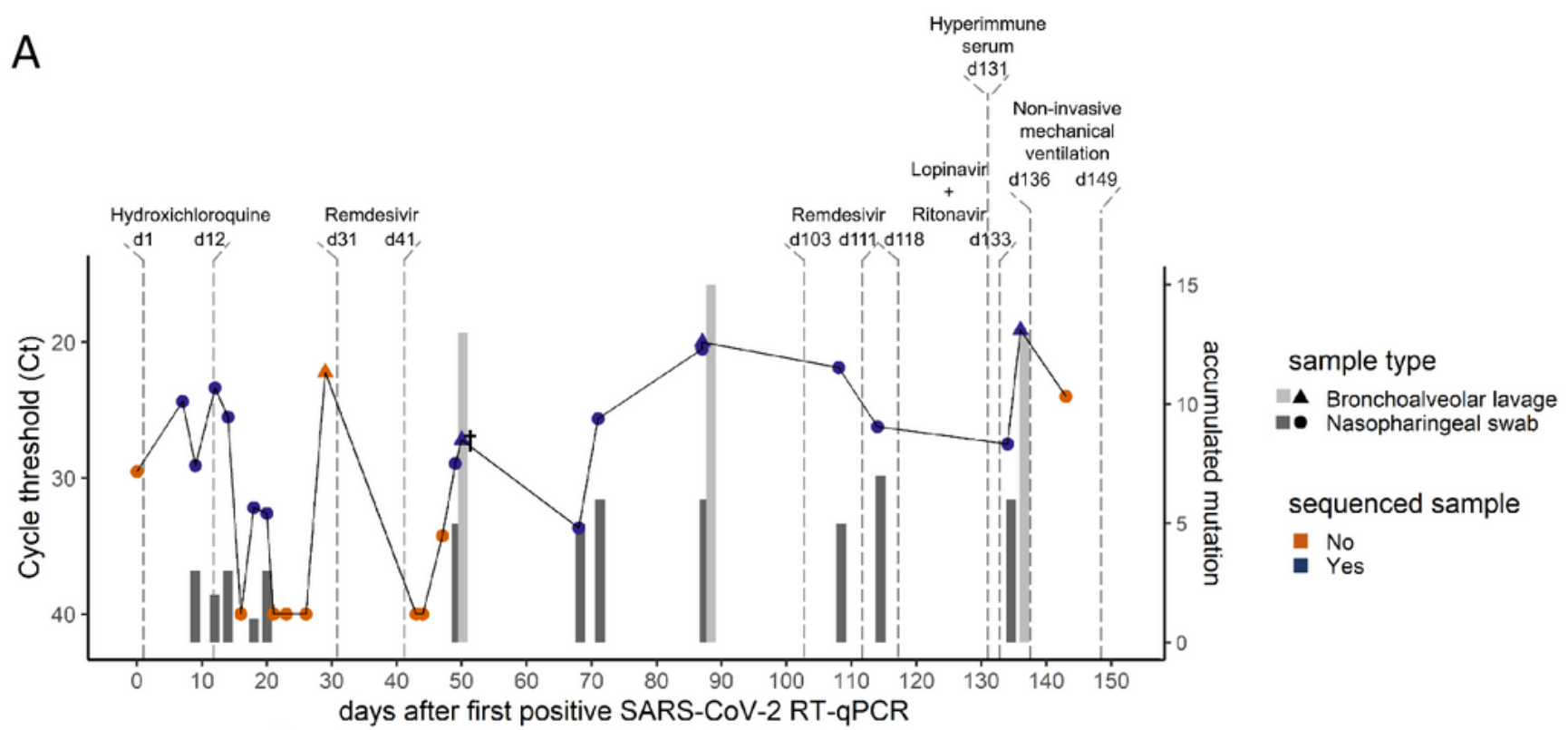

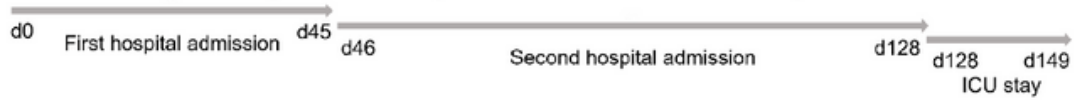
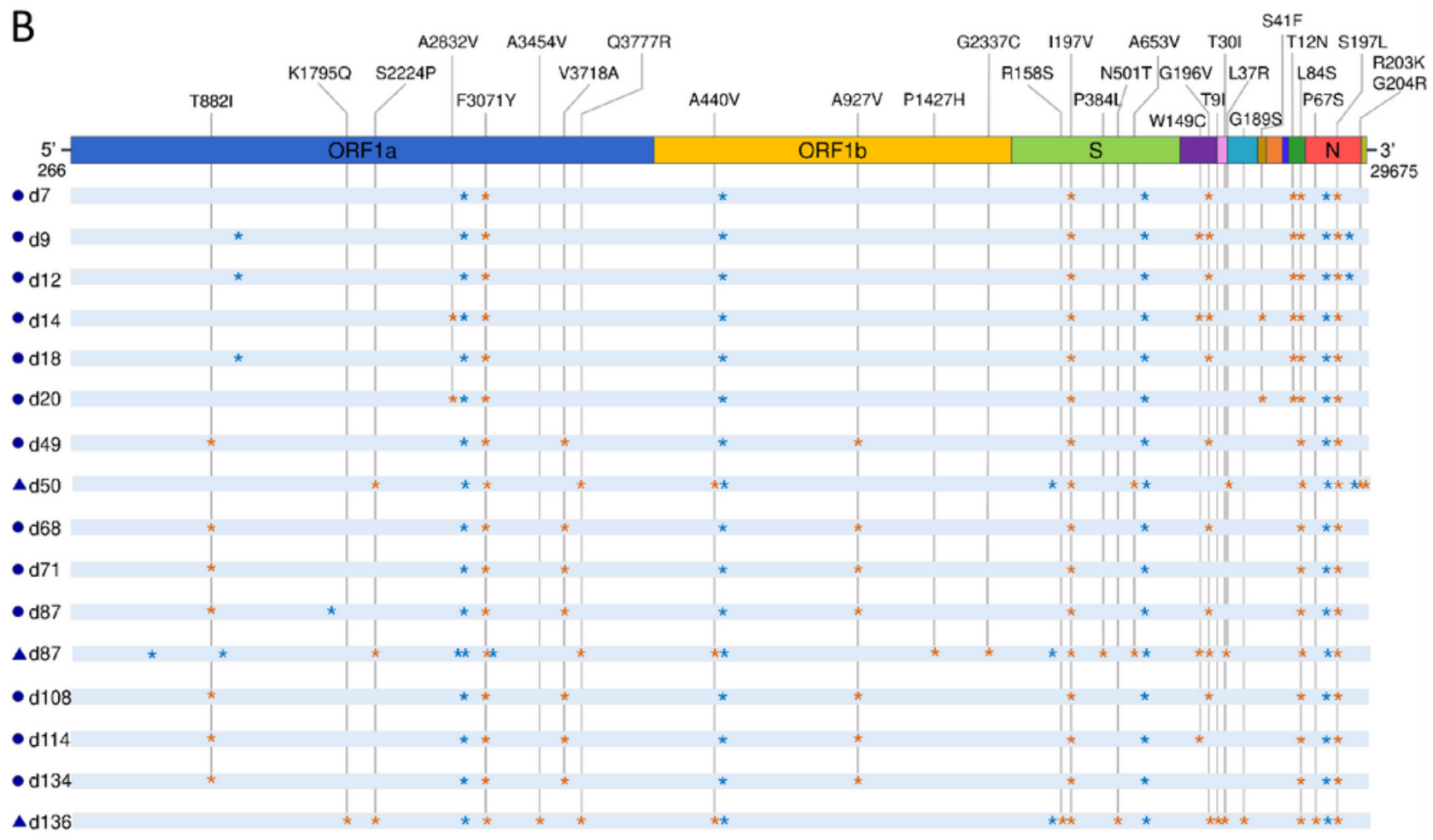

*Non-synonymous * Synonymous

\section{Figure 1}

Five-month longitudinal study of SARS-CoV-2 positive samples collected from an XLAimmunocompromised patient. A) Chronological visualization of samples collected throughout the course of infection until patient death (day 149). RT-qPCR cycle threshold (Ct) are shown for collected nasopharyngeal swab (NP, circle) and bronchoalveolar (BAL, triangle) samples, with sequenced samples highlighted in blue. Vertical bars represent the accumulated number of mutations in the sequenced 
genome compared to the consensus viral sequence obtained from the first NP sample (day 9). $†$ At day 50, a BAL sample was shown to have actively replicating SARS-CoV-2 viruses. B) Graphical representation of SARS-CoV-2 whole-genome consensus sequences with synonymous (blue asterisks) and non-synonymous mutations (orange asterisks) identified as compared to the Wuhan-Hu-1 reference sequence (NC_045512.2). Only non-synonymous mutations are identified with the amino acid changes in the figure. On day 50, in the $\mathrm{N}$ gene, the amino acid substitution S197L is replaced by S197T.

\section{Supplementary Files}

This is a list of supplementary files associated with this preprint. Click to download.

- ImmunocompromisedpatientpaperfinalpreprintSM.docx 